\title{
A TAUBERIAN THEOREM FOR $\alpha$-CONVERGENCE OF CESARO MEANS
}

\author{
A. V. BOYD
}

The object of this note is to generalize certain Tauberian results proved by Gehring [3] for summability $(C, k ; \alpha)$. The notation is as in [3], with the following additional definitions: If $k>-1$, then $A_{n}^{k}, B_{n}^{k}$ denote the $n$th Cesàro sums of order $k$ for the series $\sum_{n=0}^{\infty} a_{n}$, $\sum_{n=0}^{\infty} b_{n}$, where $b_{n}=n a_{n} . A_{n}^{-1}, B_{n}^{-1}$ denote $a_{n}, b_{n}$. Summability $(C$, $-1 ; \alpha)$ of $\sum a_{n}$ will be taken to mean summability $(C, 0 ; \alpha)$ of $\sum a_{n}$ together with the condition that sequence $\left\{n a_{n}\right\}$ be $\alpha$-convergent to 0 .

Gehring's Tauberian theorems are:

THEOREM 4.3.2. Suppose that $0 \leqq \alpha \leqq 1$ and that $\sum a_{n}$ is summable $(A ; \alpha)$ to $S$. If the sequence $\left\{n a_{n}\right\}$ is $\alpha$-convergent to $0, \sum a_{n}$ is $\alpha$-convergent to $S$.

THEOREM 4.3.3. Suppose that $0 \leqq \alpha \leqq 1$ and that $\sum a_{n}$ is summable $(A ; \alpha)$ to $S$. Then $\sum a_{n}$ is $\alpha$-convergent to $S$ if and only if the sequence $\left\{\left(a_{1}+\cdots+n a_{n}\right) / n\right\}$ is $\alpha$-convergent to 0 .

Theorem 4.3.4. Suppose that $0 \leqq \alpha \leqq 1$ and that $\sum a_{n}$ is $\alpha$-convergent. If the sequence $\left\{n a_{n}\right\}$ is $\alpha$-convergent to $0, \sum a_{n}$ is summable $(C, k ; \alpha)$ to its sum for every $k>-1$.

These will be used in the proof of the following:

THEOREM. Suppose that $0 \leqq \alpha \leqq 1$ and that $\sum a_{n}$ is summable $(A ; \alpha)$ to $S$. Then, for $r \geqq-1, \sum a_{n}$ is summable $(C, r ; \alpha)$ to $S$ if and only if the sequence $\left\{B_{n}^{r} / C_{n}^{r+1}\right\}$ is $\alpha$-convergent to 0 .

Proof of Theorem. Necessity. If $r=-1$ this follows immediately from the definition of summability $(C,-1 ; \alpha)$. If $r>-1$ then by the consistency theorem for $(C, r ; \alpha)$ summability (Gehring [3, Theorem 4.2.1]) it follows that both sequences $\left\{S_{n}^{r}\right\},\left\{S_{n}^{r+1}\right\}$ are $\alpha$-convergent to $S$. By Hardy [1, Equation (6.1.6)],

$$
S_{n}^{r}=S_{n}^{r+1}+\frac{1}{r+1} \frac{B_{n}^{r}}{C_{n}^{r+1}}
$$

and the result follows since a linear combination of sequences summable $(C, k ; \alpha)$ is itself summable $(C, k ; \alpha)$.

Received by the editors January 11, 1955 and, in revised form, March 3, 1955. 
Sufficiency. If $r>-1$ it may be shown ${ }^{1}$ as in Szász $[4, \S 1]$, that

$$
\frac{1}{y+1} \sum_{n=0}^{\infty} S_{n}^{r+1}\left(1-\frac{1}{y+1}\right)^{n}=\frac{r+1}{y} \int_{0}^{y}\left(1-\frac{u}{y}\right)^{r} \phi(u) d u,
$$

where $\phi(u)=f(1-1 /(u+1))$ and $f(x)=\sum a_{n} x^{n}$.

CASE (i). $\alpha=0, r>-1$. Since $\sum a_{n}$ is summable $(A)$ to $S$ it follows that $\phi(u)$ tends to $S$ as $u$ increases. The right-hand side of (2), being the $(r+1)$ th transform of $\phi(u)$, also tends to $S$; and so the sequence $\left\{S_{n}^{+1}\right\}$ is summable $(A ; \alpha)$ to $S$.

CAse (ii). $0<\alpha \leqq 1, r>-1$. Putting

$$
g(y)=\frac{1}{y+1} \sum_{n=0}^{\infty} S_{n}^{r+1}\left(1-\frac{1}{y+1}\right)^{n}
$$

we get, from (2), that

$$
g(y)=(r+1) \int_{0}^{1}(1-v)^{r} \phi(v y) d v
$$

where $\phi(u)$ now has bounded $\alpha$-variation over $(0, \infty)$. Let

$$
\begin{aligned}
V & =\left[\sum_{v=1}^{N}\left|g\left(y_{v}\right)-g\left(y_{v-1}\right)\right| 1 / \alpha\right] \alpha \\
& =(r+1)\left[\sum_{v=1}^{N}\left|\int_{0}^{1}(1-v)^{r}\left\{\phi\left(v y_{v}\right)-\phi\left(v y_{v-1}\right)\right\} d v\right|^{1 / \alpha}\right]^{\alpha} .
\end{aligned}
$$

Then by Theorem 201 of [5], ${ }^{2}$

$$
\begin{aligned}
V & \leqq(r+1) \int_{0}^{1}(1-v)^{r}\left\{\sum_{\nu=1}^{N}\left|\phi\left(v y_{\nu}\right)-\phi\left(v y_{\nu-1}\right)\right|^{1 / \alpha}\right\}^{\alpha} d v \\
& \leqq(r+1) M \int_{0}^{1}(1-v)^{r} d v \\
& =M
\end{aligned}
$$

where $M=V_{\alpha}\{\phi(x) ; 0 \leqq x<\infty\}$. Thus $g(y)$ has bounded $\alpha$-variation $\operatorname{over}(0, \infty)$ and so the series $\sum s_{n}$, where $s_{n}=S_{n}^{r+1}-S_{n-1}^{r+1}$, is summable $(A ; \alpha)$ to $S$. Further, by Hardy [1, Equation (6.1.6)],

$$
n s_{n}=n\left(S_{n}^{r+1}-S_{n-1}^{r+1}\right)=B_{n}^{r} / C_{n}^{r+1}
$$

\footnotetext{
1 Note however the error in Szász's equation (2.4). There, and in the previous line, occurs an extraneous term $(1+1 / y)^{\alpha-1}$.

2 I am indebted to a referee for shortening my argument at this step.
} 
so that sequence $\left\{n s_{n}\right\}$ is $\alpha$-convergent to 0 . Hence by Theorem 4.3.2 we have that $\sum s_{n}$ and sequence $\left\{S_{n}^{r+1}\right\}$ are $\alpha$-convergent to $S$.

It is readily seen from Minkowski's inequality that the sum of two $\alpha$-convergent sequences is also $\alpha$-convergent, and we therefore deduce from (1) that $\left\{S_{n}^{r}\right\}$ is $\alpha$-convergent to $S$; i.e., $\sum a_{n}$ is summable ( $C, r$; $\alpha)$ to $S$.

CASE (iii). $r=-1$. When $\alpha=0$ the result reduces to Tauber's original theorem; when $0<\alpha<1$ it follows from Theorem 4.3.2. For $\alpha=1$ the result was proved by Hyslop [2, Theorem 4].

\section{REFERENCES}

1. G. H. Hardy, Divergent series, 1949.

2. J. M. Hyslop, $A$ Tauberian theorem for absolute summability, J. London Math. Soc. vol. 12 (1937) pp. 176-180.

3. F. W. Gehring, $A$ study of $\alpha$-variation, I, Trans. Amer. Math. Soc. vol. 76 (1954) pp. $420-443$.

4. O. Szász, On products of summability methods, Proc. Amer. Math. Soc. vol. 3 (1952) pp. 257-263.

5. G. H. Hardy, J. E. Littlewood, and G. P6lya, Inequalities, 1934.

UNIVERSITY OF THE WITWATERSRAND 